\title{
Morphological variation and heritability studies of Lagenaria siceraria landraces from northern KwaZulu-Natal, South Africa
}

\author{
LUNGELO GIVEN BUTHELEZI ${ }^{1}$, SYDNEY MAVENGAHAMA ${ }^{2}$, NONTUTHUKO ROSEMARY NTULI ${ }^{1, \bullet}$ \\ ${ }^{1}$ Department of Botany, Faculty of Science and Agriculture, University of Zululand, Empangeni, Private Bag X1001, KwaDlangezwa 3886, South \\ Africa. Telp./fax.: +27 (0) 35902 6105. ‘email: NtuliR@unizulu.ac.za. \\ ${ }^{2}$ Crop Science Department, Faculty of Natural and Agricultural Sciences, School of Agricultural Sciences, North-West University, Private Bag X2046, \\ Mmabatho 2735, South Africa
}

Manuscript received: 19 February 2018. Revision accepted: 28 February 2019.

\begin{abstract}
Buthelezi LG, Mavengahama S, Ntuli NR. Morphological variation and heritability studies of Lagenaria siceraria landraces from northern KwaZulu-Natal, South Africa. Biodiversitas 20: 922-930. Lagenaria siceraria (Molina) Standley belongs to the Cucurbitaceae family. Its nutritious tender shoots, fruits, and seeds are widely consumed in rural communities as leafy vegetables. However, studies on variation and heritability of its agronomic traits are lacking. This study aimed to characterize variability and heritability among L. siceraria genotypes with various fruit and seed morphology from different origins. Seedling, vegetative and reproductive traits were compared among landraces. Landraces varied significantly in their seedling, vegetative and reproductive traits. Significant positive correlations were mainly recorded among reproductive traits. The first five informative principal components had a total variability of $74.393 \%$. Biplot and dendrogram grouped landraces mainly according to fruit and seed morphology and then their origin. In a biplot, Cluster-I grouped landraces with pear-shaped (KSP, RSP, RRP) and curvilinear-shaped (NqRC) fruits. Cluster-II mainly associated landraces with smooth and curvilinear-shaped fruits (KSC, NqSC, NSRC, NSC, MSC, and DSI) from different origins. Cluster-III associated landraces with rough-textured fruits from the same origin (NRB and NRC). High heritability estimate was recorded among fruit and seed traits. This was the first comprehensive variability and heritability study in the country and therefore it formed basis for available $L$. siceraria germplasm essential for future breeding programs.
\end{abstract}

Keywords: Heritability, Lagenaria siceraria, landraces, morphological traits

\section{INTRODUCTION}

Lagenaria siceraria (Molina) Standley, commonly known as bottle gourd is a member of the Cucurbitaceae family. It is a prostrate-growing, herbaceous and monoecious plant easily distinguished by its white flowers (Muralidharan et al. 2017). L. siceraria is believed to be of African and Asian origin (Morimoto et al. 2005). Juvenile shoots and fruits of $L$. siceraria are sliced and cooked as vegetables throughoutSouth Africa (Xaba and Croeser 2011; Sithole et al. 2015). Mature seeds are roasted and crushed to a paste used to thicken stews, while seed oil extract is an ideal alternative for vegetable oil (Chimonyo and Modi 2013). L. siceraria shows great diversity in: germination percentage, cotyledonous leaf area, seedling height, shoot growth, vine length, leaf growth, number of branches, number of leaves, shoot fresh mass, shoot dry mass, shoot moisture content (Uddin et al. 2014; Kumar 2016; Muralidharan et al. 2017). Landraces with larger seeds tend to have higher germination percentage and produce vigorously elongating seedlings with bigger cotyledonous than those with smaller seeds (Chimonyo and Modi 2013). Landraces with longer vines are less branching and have bigger and fewer leaves (Koffi et al. 2009; Mlandenovic et al. 2011), and the opposite is true for landraces with shorter vines (Uddin et al. 2014; Kumar
2016). The shape of $L$. siceraria fruit varies between pearshape, conic, oval, isodiametric, discus-shaped and curvilinear (Mlandenovic et al. 2011; Mashilo et al. 2017). Fruit color also varies from pale to dark green, with either smooth or rough fruit texture (Mashilo et al. 2016; Mashilo et al. 2017). Seeds are one of the major differentiating traits among L. siceraria landraces (Sivaraj and Pandravada 2005). There are three types of seeds, namely, siceraria, asiatica, and intermediate type (Morimoto et al. 2005). Seed color varies from creamy brown to dark brown, with either smooth or leathery texture (Mlandenovic et al. 2012). Some landraces have smooth to wavy margins with either presence of absence of prominent seed lines (Mlandenovic et al. 2012). Landraces with bigger fruits normally produce heavier seeds (Muralidharan et al. 2017). Although $L$. siceraria landraces have various utensil, medicinal and food use, they are still underutilized in South Africa, including northern KwaZulu-Natal. Studies in the morphological diversity among these landraces are still marginal in the country. Therefore, a study on their morphological diversity will benefit future breeding studies and preservation of these landraces. The aim of this study was to characterize variability among $L$. siceraria genotypes of different origins using morphological traits. 


\section{MATERIALS AND METHODS}

\section{Study area and seed collection}

Germplasm of the study consisted of $14 \mathrm{~L}$. Siceraria landraces seeds that were collected from Dundee $\left(28.1650^{\circ}\right.$ $\mathrm{S}$, 30.2343 $\mathrm{E}$ ), Khangelani $\left(29.0106^{\circ} \mathrm{S}, 31.2211^{\circ} \mathrm{E}\right)$, Mbazwana $\left(27.4937^{\circ} \mathrm{S}, 32.5882^{\circ} \mathrm{E}\right)$, Ndumo $\left(26.9342^{\circ} \mathrm{S}\right.$, $\left.32.2824^{\circ} \mathrm{E}\right)$, Nquthu $\left(28.2195^{\circ} \mathrm{S}, 30.6746^{\circ} \mathrm{E}\right)$ and Rorker's Drift $\left(28.3492^{\circ} \mathrm{S}, 30.5351^{\circ} \mathrm{E}\right)$ in northern KwaZulu-Natal. Landrace names were coined according to their origin as well as fruit and seed morphology (Table 1). The field experiment was conducted at the University of Zululand, KwaDlangezwa Campus $\left(28.8530^{\circ} \mathrm{S}, 31.8500^{\circ}\right.$ E), with a sub-tropical climate (Ntuli and Zobolo 2008). KwaDlangezwa area has a daily mean temperature of $28.4^{\circ} \mathrm{C}$ in summer and $14.5^{\circ} \mathrm{C}$ in winter (Nelson et al. 2014). This study was conducted over two summer seasons (September 2016-February 2017) and (September 2017February 2018), in a complete randomized block design.

\section{Procedures}

Eighty (80) seeds of each of the 14 landraces were sown onto the plug trays and irrigated to plug tray capacity two to three times per week. At 31 days after sowing (31 DAS) all landraces where at first to second true leaf stage and were transplanted onto the field. The field was divided into 14 equal sized plots with three replicates per landrace which amounted to 42 plots in total. Each plot was $9 \mathrm{~m}^{2}$ with $2 \mathrm{~m}$ inter-row spacing and $1 \mathrm{~m}$ intra-row spacing, housing 16 plants per plot. This had a total of 672 plants in a $1050 \mathrm{~m}^{2}$ plot of land. At transplanting, fertilizer NPK 2: 3: 4 (30) at a rate of $40 \mathrm{~g} / \mathrm{m}^{2}$ was applied below the seedlings in $10-15 \mathrm{~cm}$ deep pits and the field was irrigated to field capacity. At 31 days after transplant (DAT), nitrogen fertilizer (Limestone Ammonium Nitrate (LAN 28) of the same rate was band placed around each plant. Plants were irrigated adequately depending on the amount of rainfall and temperature. Weeding and insecticides applications were performed when necessary. Seedling traits [germination percentage $(\%)$, seedling height $(\mathrm{mm})$ and cotyledonous leaf area $\left(\mathrm{mm}^{2}\right)$ ] were measured at 31 DAS while still growing in the plug trays. All landraces were at a vining stage (with a minimum of six leaves) at 38 DAT. Shoot and leaf growth percentages were determined from 38 to 45 DAT. Vegetative traits: vine length $(\mathrm{m})$, number of branches, number of leaves, total chlorophyll content $(\mathrm{g} / \mathrm{mL})$, shoot fresh mass $(\mathrm{g})$, shoot dry mass $(\mathrm{g})$, shoot moisture content $(\%)$, tendril basal length $(\mathrm{mm})$, tendril longer lobe length $(\mathrm{mm})$ and tendril shorter lobe length $(\mathrm{mm})$ were measured at 45 DAT. Flower traits were recorded at $60 \mathrm{DAT}$. At harvest (125 DAT) the following fruit and seed traits were measured: number of fruits per plot, fruit yield per plot $(\mathrm{kg} / \mathrm{ha})$, fruit mass $(\mathrm{kg})$, fruit length $(\mathrm{mm})$, fruit width $(\mathrm{mm})$, pedicel length $(\mathrm{mm})$, fruit rind thickness $(\mathrm{mm})$, seed length $(\mathrm{mm})$, seed width $(\mathrm{mm}), 100$ seed mass ( $\mathrm{g}$ ) and total seed mass $(\mathrm{g})$. Seedling, vegetative and fruit traits were measured from nine plants (replicates) per landrace.

\section{Data analysis}

Data were subjected to ANOVA using the GenStat $15^{\text {th }}$ edition. Means were separated using Tukey's LSD at 5\% significant level. Correlations and Principal Component Analysis (PCA) were implemented to determine multicharacter variation. Cluster Analysis through biplot and dendrogram was conducted to study the similarities and dissimilarities of landraces from different origins.

\section{Estimation of variance components}

The phenotypic, genotypic and environmental variances and coefficient of variation were calculated according to the formula described by Burton and Devane (1953) and cited by Singh et al. (2017) as follows:

Environmental variance $\left(\delta^{2} \mathrm{e}\right)=\mathrm{MSE}$

Genotypic variance $\left(\delta^{2} \mathrm{~g}\right)$

$\delta^{2} \mathrm{~g}=\frac{(M S G-M S E)}{r}$

Phenotypic variance $\left(\delta^{2} \mathrm{p}\right)$

$\delta^{2} p=\delta^{2} g+\delta^{2} e$

Where:

MSG : Square due to genotype

MSE : Square of error (environmental variance)

$r \quad$ : Number of replications

Phenotypic coefficient of variation $(\mathrm{PCV})=$

$$
\frac{\sqrt{\delta^{2} g}}{X} \times 100
$$

Genotypic coefficient of variation $(\mathrm{GCV})=$

$$
\frac{\sqrt{\delta^{2} \mathrm{p}}}{X} \times 100
$$

Where

$\delta^{2} \mathrm{p} \quad$ : Phenotypic variation

$\delta^{2} \mathrm{~g}:$ Genotypic variation

$\mathrm{X}$ : Grand mean of the character studied

Estimation of heritability in broad sense: Broad-sense heritability $\left(\mathrm{h}^{2}\right)$ expressed as the percentage of the ratio of the genotypic variance $\left(\delta^{2} \mathrm{~g}\right)$ to the phenotypic variance $\left(\delta^{2} \mathrm{p}\right)$, according to Allard (1960), was calculated with the following formula:

$$
h^{2}=\frac{\delta^{2} \mathrm{~g}}{\delta^{2} \mathrm{p}} \times 100
$$

Genetic advance (GA) was estimated as per formula given by Allard (1960) and cited by Meena et al. (2015).

$$
\mathrm{GA}=\mathrm{k} \times \sqrt{\frac{\delta^{2} \mathrm{px}}{\delta^{2}}} \quad \sqrt{\frac{\delta^{2} \mathrm{px}}{\delta^{2}}}
$$

Where:

$\delta^{2} \mathrm{p} \quad$ : Phenotypic variation

$\delta^{2} \mathrm{~g}$ : Genotypic variation

$\mathrm{k} \quad$ : The standard selection differential at $5 \%$ selection intensity $(\mathrm{k}=2.063)$ 


\section{RESULTS AND DISCUSSION}

\section{Seedling traits}

Landrace DSI from Dundee had the highest (95\%) germination percentage whereas landrace RRP from Rorke's Drift had the least (67.5\%) with an average of $83 \%$ (Table 2). Chimonyo and Modi (2013) reported a lower range of 15 to $85 \%$ with an average of $47.2 \%$ among landraces of different origins. Landraces with similar fruit morphology but different origins differed in their germination percentage. This was evident in smooth pearshaped landraces: KSP from Khangelani and RSP from Rorke's Drift as well as smooth curvilinear-shaped landraces: KSC from Khangelani, MSC from Mbazwana and NSC from Nquthu. Diversity in origin may be responsible for variations between the phenotypic and genotypic coefficient of variation for germination percentage (Table 6). Variation in phenotypic and genotypic coefficient of variation in traits result from the diversity in the origin of landraces (Jain et al. 2017). Landrace KSC from Khangelani had the tallest seedlings (48.8 $\mathrm{mm}$ ) but landrace NRB from Ndumo had the shortest $(22.5 \mathrm{~mm})$, with an average seedling height of $37.4 \mathrm{~mm}$ (Table 2). Yetisir et al. (2008) reported a similar seedling height range of 22-56 $\mathrm{mm}$ with an average of $39 \mathrm{~mm}$ from accessions collected in Turkey. Landrace NSRC had the largest cotyledonous leaf area $\left(1726 \mathrm{~mm}^{2}\right)$ while landrace NRC had the smallest $\left(880 \mathrm{~mm}^{2}\right)$ with an average of 1289 $\mathrm{mm}^{2}$. Mashilo et al. (2016) reported a lower cotyledonous leaf area range (404.48-1484.04 $\left.\mathrm{mm}^{2}\right)$ and average (868.17 $\mathrm{mm}^{2}$ )obtained among $L$. siceraria landraces. In the current study, landraces with larger and heavier seeds (KSP, KSC, RSP, NqSC, NRC, NSRP, NSRC and DSI) produce superior seedlings, which is similar to the findings of Chimonyo and Modi (2013). This is probably due to a prolonged starch and energy provision period during the seed embryonic developmental stage (Chimonyo and Modi 2013).

\section{Vegetative traits}

Landrace KSC had the most vigorous shoot growth (1256\%) and NSRP had the least (412\%), with an average of $889 \%$ (Table 2). Yetisir et al. (2008) reported a lower shoot growth ranging from $180-500 \%$ with an average of $332.5 \%$ on Citrullus lanatus. Landraces of the current study propagated from north to south direction had a leaf growth ranging from 1518-9566\% with an average of 5281\%. A lower L. siceraria leaf growth average of $298.9 \%$ was recorded from 45-55 DAS in India by Sharma and Tomar (2016). The vine length ranged from 2.3-4.0 $\mathrm{m}$ with a mean of $2.93 \mathrm{~m}$. Mlandenovic et al. (2012) reported similar findings, recording a vine length range of 2.8-3.2 $\mathrm{m}$ with an average of $3.1 \mathrm{~m}$. Landrace KRI recorded the highest number of branches (8.4) while MSC had the least (4.4), with an average of 6.1 branches. A higher range of 9.4 to 34.1 branches with a mean of 19.2 was recorded by Mashilo et al. (2016). The landrace KRI from Khangelani had the most numerous leaves per plant (41.5) but NSC from Nquthu had the least number of leaves (17.6), with an average of 29.7 leaves. C. lanatus had a higher average leaf number at 36.33 (Rahaman et al. 2018). The difference in genera and subjection of $C$. lanatus seeds to mutagens explains the higher number of leaves (Rahaman et al. 2018).

Landrace RRP had the highest total chlorophyll content $(16.6 \mathrm{~g} / \mathrm{ml})$ butNSRP had the lowest $(11.7 \mathrm{~g} / \mathrm{ml})$, with an average of $14.5 \mathrm{~g} / \mathrm{ml}$. Wang et al. (2009) recorded a lower chlorophyll content mean of $0.016 \mathrm{mg} / \mathrm{gon}$ Cucumis sativus. Koppad et al. (2016) also reported a lower total chlorophyll content range $\left(0.46-2.39 \mathrm{mg} / \mathrm{cm}^{2}\right)$ with a mean of $1.44 \mathrm{mg} / \mathrm{cm}^{2}$. The tendril basal length ranged from $28.4-$ $75 \mathrm{~mm}$ with a mean of $53.3 \mathrm{~mm}$; tendril longer lobe length ranged from 162.4-267.6 mm with a mean of $211.2 \mathrm{~mm}$ and tendril shorter lobe length ranged from 88.1-167.5 mm with a mean of $125.4 \mathrm{~mm}$. Deepthi et al. (2016) and Koppad et al. (2016)reported longer tendrils ranging from 190.3-364.4 mm and241.7-308.3 mm, respectively. Shoot fresh mass ranged from 35.1-59.5 $\mathrm{g}$ with a mean of $53.9 \mathrm{~g}$; shoot dry mass ranged from 8.1-12.5 $\mathrm{g}$ with an average of $10.8 \mathrm{~g}$ and the shoot moisture content ranged from 75.5$82.7 \%$ with a mean of $79.6 \%$. Mohammed et al. (2017) reported a higher shoot fresh mass average of $374.8 \mathrm{~g}$; shoot dry mass average of $64.7 \mathrm{~g}$ and a shoot moisture content of $82.7 \%$.

\section{Reproductive traits}

The number of staminate flowers ranged from 7.7-21.0 with a mean of 12.9 and pistillate flowers ranged from 7.021.0 with a mean of 13.2 (Table 3). Jain et al. (2017) recorded a lower number of staminate flowers ranging from 5.3-10.37 with a mean of 7.4 and pistillate flowers ranging from 6.2-15.6 and averaging 10.88. The flower sex ratio ranged from 0.4-1.6 with a mean of 1.1. Rani and Reddy (2017) reported a lower flower sex ratio ranging from 0.42 0.59 with a mean of 0.48 possibly due to lower temperatures as the study was conducted in winter. Crops from the Cucurbitaceae family have better flower development in warmer seasons compared to colder seasons where flower development is delayed and there is a high fruit abortion rate (Korkmaz and Dufault 2001).

\section{Fruit yield and traits at harvest}

Landrace KSP from Khangelani at $82500 \mathrm{~kg} / \mathrm{ha}$ had the highest fruit yield per plot and MSC had the lowest at $17111 \mathrm{~kg} / \mathrm{ha}$ with a mean of $43674 \mathrm{~kg} / \mathrm{ha}$ (Table 3). Uddin et al. (2014) reported a higher fruit yield per plot ranging from $33100-82000 \mathrm{~kg} / \mathrm{ha}$ with a mean of $52300 \mathrm{~kg} / \mathrm{ha}$. Landrace RSP $(5.1 \mathrm{~kg})$ had the heaviest fruit mass and NRC had the lightest $(1.0 \mathrm{~kg}$ ) with an average of $2.0 \mathrm{~kg}$. Rani and Reddy (2017) reported a lower fruit mass range $(1.55-2.73 \mathrm{~kg})$ with an average of $2.0 \mathrm{~kg}$. The fruit length ranged from 198.0-447.0 mm with a mean of $319.0 \mathrm{~mm}$; fruit width ranged from 165.6-372.1 mm with a mean of $243.0 \mathrm{~mm}$; pedicel length ranged from 59.3-115.3 $\mathrm{mm}$ with a mean of $78.0 \mathrm{~mm}$ and fruit rind thickness ranged from 3.0-8.0 mm with a mean of $5.0 \mathrm{~mm}$. Mashilo et al. (2016) reported shorter fruits $(95.0-379.9 \mathrm{~mm})$ with a mean of $248.0 \mathrm{~mm}$; narrower fruits $(65.9-131.2 \mathrm{~mm})$ with an average of $94.8 \mathrm{~mm}$; shorter pedicels $(8.4-57.8 \mathrm{~mm})$ with a 
mean of $29.5 \mathrm{~mm}$ and thinner fruit rinds $(1.2-4.3 \mathrm{~mm})$ with a mean of $2.5 \mathrm{~mm}$.

\section{Seed size and mass}

Seed length ranged from 14.0-23.0 mm with a mean of $17.6 \mathrm{~mm}$; Seed width ranged from 4.0-14.9 mm with a mean of $9.7 \mathrm{~mm}$ and 100 seed mass ranged from 11.6-21.6 $\mathrm{g}$ with a mean of $15.6 \mathrm{~g}$ (Table 3). Mashilo et al. (2016) recorded shorter seeds ranging from 11.3-18.8 $\mathrm{mm}$ with a mean of $14.4 \mathrm{~mm}$; narrower seed width ranging from 5.0$8.7 \mathrm{~mm}$ with a mean of $6.7 \mathrm{~mm}$ and lighter 100 seed mass ranging from $7.6-23.9 \mathrm{~g}$ with a mean of $13.2 \mathrm{~g}$.

\section{Correlation}

The number of branches correlated positively with the vine length $(r=0.614)$, number of leaves $(r=0.678)$ and number of fruits per plot $(\mathrm{r}=0.673)$ (Table 3.5). This suggests that the selection of genotypes with numerous branches will instinctively lead to genotypes with longer vines, many leaves and produce many fruits per plots. Sultana et al. (2017) reported similar correlations between the number of branches, number of fruits per plot $(\mathrm{r}=$ $0.868)$ and total fruit yield $(r=0.833)$. Seed width correlated positively with all tendril traits $(\mathrm{r}=0.622$, $\mathrm{r}=$ 0.694 and $r=0.624)$ respectively. Total seed weight correlated positively with tendril basal length $(r=0.609)$. The number of staminate flowers per plot positively correlated with the number of pistillate flowers per plot $(\mathrm{r}=$ $0.781)$ and the number of fruits per plot $(r=0.613)$. Ntuli et al. (2017) reported that the number of staminate flowers of Cucurbita maxima correlated negatively to the number of pistillate flowers $(r=-0.371)$. Fruit yield per plot correlated positively with the fruit mass $(r=0.768)$ and fruit width $(r$ $=0.702)$. Thakur et al. (2017) recorded similar results, fruit mass positively correlated with fruit yield per plot at $(\mathrm{r}=$ 0.777). The fruit mass strongly correlated positively with the fruit width $(r=0.916)$. Sultana et al. (2017) also reported similar results as the fruit width is positively correlated with the fruit mass $(r=0.347)$. Hundred seed mass strongly correlated positively with the total seed mass $(\mathrm{r}=0.966)$ and they both positively correlated with fruit traits; fruit mass $(r=0.780$ and $r=0.799)$ and width $(r=$ 0.665 and $r=0.732$ ), respectively. Mlandenovic et al. (2012) reported hundred seed mass positively correlated with; total seed mass $(r=0.49)$ and fruit mass $(r=0.53)$. Hundred seed mass positively correlated with fruit width ( $\mathrm{r}$ $=0.358$ ) (Sultana et al. 2017). A study by Ntuli et al. (2017) on Cucurbita maxima, reported hundred seed mass strongly correlating positively with the total seed mass ( $\mathrm{r}=$ $0.985)$.

\section{Principal component analysis}

The principal component analysis identified diversity among landraces (Table 5). The morphological traits of the current study; shoot fresh mass, shoot dry mass, tendril basal length, tendril shorter lobe length, fruit mass, fruit width, seed length, seed width, 100 seed mass, and total seed mass are strongly positively correlated with the first principal component (PC1). The vine length, number of branches and number of fruits per plot strongly correlated negatively with PC1. These traits represent $26.635 \%$ of the total variability. Morphological traits; seedling height, cotyledonous leaf area, shoot fresh mass, the number of staminate flowers per plot and the number of pistillate flowers per plot is strongly positively correlated with the second principal component (PC2). Fruit rind thickness strongly correlated negatively with PC2.

Table 1. Description of landraces according to their origin as well as fruit and seed morphology

\begin{tabular}{|c|c|c|c|c|c|c|c|c|c|c|}
\hline $\mathbf{L R}$ & Area & $\begin{array}{l}\text { Fruit } \\
\text { color }\end{array}$ & $\begin{array}{c}\text { Fruit } \\
\text { texture }\end{array}$ & $\begin{array}{l}\text { Fruit } \\
\text { shape }\end{array}$ & Seed type & Seed color & $\begin{array}{c}\text { Seed } \\
\text { texture }\end{array}$ & $\begin{array}{l}\text { Seed } \\
\text { size }\end{array}$ & $\begin{array}{l}\text { Seed } \\
\text { line }\end{array}$ & Seed shape \\
\hline KSP & Khangelani & Pale green & Smooth & Pear & Asiatica & Brown & Leathery & Large & Present & Slightly oblong to rectangular \\
\hline $\mathrm{KSC}$ & Khangelani & Pale green & Smooth & Curvilinear & Asiatica & Brown & Leathery & Large & Present & Slightly oblong to rectangular \\
\hline KRI & Khangelani & Green & Rough & Isodiametric & Siceraria & Dark brown & Leathery & Large & Present & Slightly oblong to rectangular \\
\hline NRB & Ndumo & Dark green & Rough & Cylindrical & Siceraria & Dark brown & Smooth & Small & Absent & Oblong \\
\hline NRC & Ndumo & Dark green & Rough & Cylindrical & Siceraria & $\begin{array}{l}\text { Creamy } \\
\text { brown }\end{array}$ & Smooth & Small & Absent & Oblong \\
\hline RSP & Rorke's drift & Pale green & Smooth & Pear & Asiatica & Light brown & Leathery & Large & Present & Rectangular \\
\hline RRP & Rorke's drift & Pale green & Rough & Pear & Asiatica & Light brown & Leathery & Large & Present & Rectangular \\
\hline $\mathrm{NqRC}$ & Nquthu & Pale green & Rough & Curvilinear & Intermediate & Light brown & Leathery & Medium & Present & Slightly oblong \\
\hline NSRP & Nquthu & Pale green & Semi-rough & Pear & Intermediate & Brown & Leathery & Medium & Present & Slightly oblong \\
\hline $\mathrm{NqSC}$ & Nquthu & Pale green & Smooth & $\begin{array}{l}\text { Semi- } \\
\text { curvilinear }\end{array}$ & Asiatica & Light brown & Leathery & Medium & Present & Slightly oblong \\
\hline NSC & Nquthu & Pale green & Smooth & Curvilinear & Asiatica & Light brown & Leathery & Medium & Present & Slightly oblong \\
\hline DSI & Dundee & Dark green & Smooth & Isodiametric & Siceraria & Dark brown & Smooth & Large & Present & Oblong \\
\hline
\end{tabular}

Note: LR-Landraces-from Khangelani area with smooth, (KSP) pear-shaped; smooth, (KSC) curvilinear shape; and (KRI) rough isodiametric shape fruits; from Ndumo area (NRB) with rough fruits with brown seeds and (NRC) rough fruits with creamy seeds; from Mbazwana area (MSC) with smooth cylindrical-shape; from Rorke's Drift area (RSP) with smooth pear-shaped and (RRP) rough pearshaped; from Nquthu area (NqRC) with rough curvilinear shape, (NSRP) with semi-rough pear-shaped, (NqSC) and (NSC) with smooth curvilinear shaped and (NSRC) semi-rough curvilinear shaped and from Dundee area (DSI) with smooth isodiametric shape 
Table 2. Variation in seedling traits (31 DAS), shoot and leaf growth (38-45 DAT) and other vegetative traits (45 DAT) among Lagenaria siceraria different landraces

\begin{tabular}{|c|c|c|c|c|c|c|c|c|c|c|c|c|c|c|c|}
\hline LR & GP & SH & CLA & SG & LG & VL & NOB & NOL & TCC & TBL & TLL & TSHL & FSM & DSM & SMC \\
\hline KSP & $30.0 \mathrm{e}$ & $41.9 \mathrm{bcd}$ & $1322 \mathrm{de}$ & $1029 \mathrm{~b}$ & $5548 \mathrm{bc}$ & $2.4 \mathrm{ef}$ & $5.3 \mathrm{~cd}$ & $24.8 \mathrm{de}$ & $16.2 \mathrm{a}$ & 53.1 bcde & $171.2 \mathrm{gh}$ & $130.8 \mathrm{bcd}$ & $57.0 \mathrm{~b}$ & $7.9 \mathrm{c}$ & $32.7 \mathrm{a}$ \\
\hline $\mathrm{KSC}$ & $32.5 \mathrm{de}$ & $18.8 \mathrm{a}$ & $1306 \mathrm{def}$ & $1256 \mathrm{a}$ & $7566 \mathrm{a}$ & $3.1 \mathrm{c}$ & $5.5 \mathrm{c}$ & $39.4 \mathrm{a}$ & $13.5 \mathrm{abc}$ & $59.4 \mathrm{~b}$ & & & $59.5 \mathrm{a}$ & $12.5 \mathrm{a}$ & $79.0 \mathrm{de}$ \\
\hline KRI & $75.0 \mathrm{f}$ & $37.3 \mathrm{def}$ & $1111 \mathrm{efg}$ & $759 \mathrm{bc}$ & $7264 \mathrm{~b}$ & $3.0 \mathrm{~cd}$ & $3.4 \mathrm{a}$ & $41.4 \mathrm{a}$ & $14.5 \mathrm{abc}$ & $56.9 \mathrm{bc}$ & $189.6 \mathrm{efgh}$ & $115.6 \mathrm{de}$ & $56.5 \mathrm{bc}$ & $11.8 \mathrm{~b}$ & $79.1 \mathrm{de}$ \\
\hline NRB & $70.0 \mathrm{~b}$ & $22.5 \mathrm{~h}$ & $387 \mathrm{~g}$ & $327 \mathrm{~d}$ & 5176 cde & $3.0 \mathrm{~cd}$ & $7.6 \mathrm{~b}$ & $26.9 \mathrm{bcd}$ & $15.3 \mathrm{ab}$ & $28.8 \mathrm{~g}$ & $162.4 \mathrm{~h}$ & & .4 ef & $3.1 \mathrm{e}$ & 79.4 cde \\
\hline NRC & $30.0 \mathrm{e}$ & $40.9 \mathrm{bcd}$ & $380 \mathrm{~g}$ & $596 \mathrm{f}$ & 3726 e & $3.7 \mathrm{ab}$ & $7.8 \mathrm{ab}$ & $31.3 \mathrm{~b}$ & 14.7 & 53.8 bcde & $176.0 \mathrm{gh}$ & 8 ef & $.1 \mathrm{f}$ & $3.7 \mathrm{~d}$ & $75.2 \mathrm{~g}$ \\
\hline MSC & $30.0 \mathrm{e}$ & 37.8 & $1444 \mathrm{bcd}$ & 1594 ef & $4673 \mathrm{de}$ & $3.4 \mathrm{bc}$ & $7.4 \mathrm{de}$ & $26.3 \mathrm{~cd}$ & $14.3 \mathrm{a}$ & 75.0 & 214.8 & $135.6 \mathrm{bc}$ & $56.4 \mathrm{bc}$ & $10.5 \mathrm{c}$ & $31.3 \mathrm{a}$ \\
\hline RSP & 75.0 & 35.0 & 1049 & $362 \mathrm{bc}$ & 3888 e & $2.4 \mathrm{f}$ & $4.9 \mathrm{de}$ & $25.8 \mathrm{~cd}$ & $13.8 \mathrm{abc}$ & $59.1 \mathrm{a}$ & 267 & & $59.1 \mathrm{a}$ & $12.5 \mathrm{a}$ & $78.9 \mathrm{e}$ \\
\hline RRP & $57.5 \mathrm{~g}$ & $30.9 \mathrm{~g}$ & $1017 \mathrm{~g}$ & $740 \mathrm{e}$ & $5325 \mathrm{bcd}$ & $2.4 \mathrm{f}$ & $4.6 \mathrm{de}$ & $27.3 \mathrm{bcd}$ & $16.6 \mathrm{a}$ & $75.0 \mathrm{a}$ & 267. & bcd & $58.1 \mathrm{ab}$ & $11.7 \mathrm{~b}$ & $30.0 \mathrm{c}$ \\
\hline $\mathrm{NqRC}$ & $90.0 \mathrm{~b}$ & $33.5 \mathrm{fg}$ & $1118 \mathrm{efg}$ & $1084 \mathrm{~b}$ & $5287 \mathrm{bcd}$ & $2.3 \mathrm{f}$ & $7.1 \mathrm{~b}$ & $25.8 \mathrm{~cd}$ & $12.7 \mathrm{bc}$ & 54.1 bcde & 256. & & $54.1 \mathrm{c}$ & $10.5 \mathrm{c}$ & $30.5 \mathrm{bc}$ \\
\hline NSRP & $90.0 \mathrm{~b}$ & $33.1 \mathrm{bc}$ & $1627 \mathrm{abc}$ & $+12 \mathrm{~g}$ & 4620 de & $4.0 \mathrm{a}$ & $7.4 \mathrm{~b}$ & $39.6 \mathrm{a}$ & $11.7 \mathrm{c}$ & 50.0 & 226.0 & & $52.7 \mathrm{~cd}$ & $9.7 \mathrm{c}$ & $31.5 \mathrm{a}$ \\
\hline $\mathrm{NqSC}$ & $32.5 \mathrm{de}$ & $14.4 \mathrm{ab}$ & $1443 \mathrm{bcd}$ & $11098 \mathrm{~b}$ & $4002 \mathrm{e}$ & $2.2 \mathrm{f}$ & $5 \mathrm{cde}$ & $21.0 \mathrm{ef}$ & $15.8 \mathrm{ab}$ & $54.8 \mathrm{bcd}$ & 238.4 & & $59.0 \mathrm{ab}$ & $12.1 \mathrm{ab}$ & $79.4 \mathrm{~d}$ \\
\hline NSC & $35.0 \mathrm{c}$ & $31.4 \mathrm{~g}$ & $1422 \mathrm{~cd}$ & $393 \mathrm{~cd}$ & 5448 bcde & $2.6 \mathrm{def}$ & $4.6 \mathrm{de}$ & $17.6 \mathrm{e}$ & $13.5 \mathrm{abc}$ & $57.5 \mathrm{bc}$ & 219.6 & cde & $59.2 \mathrm{ab}$ & $12.1 \mathrm{a}$ & $76.4 \mathrm{f}$ \\
\hline NSRC & $32.5 \mathrm{de}$ & $39.4 \mathrm{cde}$ & $1726 \mathrm{a}$ & $770 \mathrm{bc}$ & $1518 \mathrm{f}$ & $3.8 \mathrm{ab}$ & $7.5 \mathrm{~b}$ & $38.5 \mathrm{a}$ & $15.0 \mathrm{abc}$ & $45.9 \mathrm{def}$ & $182.5 \mathrm{f}$ & de & $51.1 \mathrm{~d}$ & $7.9 \mathrm{c}$ & $30.6 \mathrm{~b}$ \\
\hline DSI & $75.0 \mathrm{a}$ & $+3.1 \mathrm{bc}$ & $1695 \mathrm{ab}$ & $325 \mathrm{bc}$ & 4893 cde & $2.9 \mathrm{cde}$ & $4.8 \mathrm{de}$ & $29.9 \mathrm{bc}$ & $16.1 \mathrm{a}$ & $43.0 \mathrm{f}$ & $201.0 \mathrm{defg}$ & $111.5 \mathrm{de}$ & $56.8 \mathrm{bc}$ & $11.0 \mathrm{c}$ & $30.6 \mathrm{~b}$ \\
\hline Mean & 32.5 & 37.4 & 1288 & 389 & 5281 & 2.9 & 5.1 & 29.7 & 14.4 & 53.3 & 211.3 & 125.4 & 53.9 & 10.8 & 79.6 \\
\hline P value & J.404 & $<.001$ & $<.001$ & $<.001$ & $<.001$ & $<.001$ & $<.001$ & $<.001$ & $<.001$ & $<.001$ & $<.001$ & $<.001$ & $<.001$ & $<.001$ & $<.001$ \\
\hline LSD & 13.92 & 4.87 & 268.8 & 47.83 & 1866.8 & 0.54 & 0.72 & 7.41 & 0.003 & 7.2 & 33.38 & 20.00 & 1.62 & 0.54 & 0.76 \\
\hline $\mathrm{zV} \%$ & 29.5 & 13.1 & 21.0 & 54.2 & 35.6 & 18.5 & 12.0 & 15.0 & 24.1 & 17.5 & 15.9 & 16.1 & 1.8 & 3.0 & 0.6 \\
\hline
\end{tabular}

Note: LR-Landraces. Description of landraces is in Table 1. Variables; GP-Germination percentage, CLA-Cotyledonous leaf area (mm), SH-Seedling height (mm), SG-Shoot growth $(\%)$ and VL-Vine length (m), LG-Leaf growth (\%), NOB-Number of branches, NOLNumber of leaves, TCC-Total chlorophyll content (g/mL X10-3), SFM-Shoot fresh mass (g), SDM-Shoot dry mass (g), SMC-Shoot moisture content $(\%)$, TBL-Tendril basal length $(\mathrm{mm})$, TLL-Tendril longer lobe length $(\mathrm{mm})$, TSHL-Tendril shorter lobe length (mm). Mean values within a column followed by a different letter (s) differ significantly $\mathrm{p}<0.05$ according to the multiple range test.

Table 3. Diversity in flower, fruit and seed traits among Lagenaria siceraria landraces

\begin{tabular}{|c|c|c|c|c|c|c|c|c|c|c|c|c|c|c|}
\hline LR & NSF/P & NPF/p & FSR & NF/p & FY/p & FM & FL & FW & PL & FRT & SL & SW & 100SM & TSM \\
\hline KSP & 13.3 cde & $18.3 \mathrm{ab}$ & $.7 \mathrm{def}$ & $5.0 \mathrm{~b}$ & $82500 \mathrm{a}$ & $4.95 \mathrm{a}$ & $417.9 \mathrm{~b}$ & $47.1 \mathrm{~b}$ & 78.6 cde & $7.5 \mathrm{~b}$ & $15.8 \mathrm{hi}$ & $.5 \mathrm{e}$ & $21.6 \mathrm{a}$ & $86.8 \mathrm{~b}$ \\
\hline $\mathrm{KSC}$ & $8.3 \mathrm{~g}$ & bcd & 6 ef & $5.7 \mathrm{~b}$ & $763 \mathrm{bc}$ & $1.48 \mathrm{e}$ & $9.2 \mathrm{de}$ & $2 d$ & 71.0 defg & $3.9 \mathrm{f}$ & 7.4 ef & 5 e & & $2.3 \mathrm{~d}$ \\
\hline KRI & $21.0 \mathrm{a}$ & $18.7 \mathrm{ab}$ & & $3 \mathrm{a}$ & $9 a b$ & $1.46 \mathrm{ef}$ & $0.7 \mathrm{j}$ & & $62.9 \mathrm{fg}$ & $8.0 \mathrm{a}$ & $15.5 \mathrm{i}$ & 0 ef & $2 \mathrm{~cd}$ & $53.1 \mathrm{~d}$ \\
\hline NRB & $7.7 \mathrm{~g}$ & $7.3 \mathrm{e}$ & $1.2 \mathrm{bc}$ & $25.3 \mathrm{ab}$ & $29500 \mathrm{bc}$ & $1.05 \mathrm{fg}$ & $197.9 \mathrm{k}$ & & $75.0 \mathrm{def}$ & $7.0 \mathrm{c}$ & $14.0 \mathrm{j}$ & $6.5 \mathrm{~h}$ & & $31.7 \mathrm{f}$ \\
\hline NRC & $9.0 \mathrm{fg}$ & $9.7 \mathrm{de}$ & $1.0 \mathrm{~cd}$ & $23.3 \mathrm{ab}$ & 24889 bc & $0.96 \mathrm{~g}$ & & & & $6.4 \mathrm{~d}$ & $16.6 \mathrm{fgh}$ & $7.8 \mathrm{f}$ & & $29.5 \mathrm{f}$ \\
\hline MSC & $9.3 \mathrm{fg}$ & $10.0 \mathrm{de}$ & $1.5 \mathrm{a}$ & $14.0 \mathrm{~b}$ & & $1.11 \mathrm{efg}$ & $380.7 \mathrm{c}$ & & $80.5 \mathrm{cde}$ & $4.3 \mathrm{f}$ & 16.4 ghi & $7.1 \mathrm{~g}$ & $2 \mathrm{de}$ & $41.2 \mathrm{e}$ \\
\hline RSP & 11.0 efg & $7.7 \mathrm{e}$ & $1.3 \mathrm{bc}$ & $11.0 \mathrm{~b}$ & $62334 a b c$ & $5.11 \mathrm{a}$ & $340.0 \mathrm{def}$ & $372.1 \mathrm{a}$ & & $6.0 \mathrm{~d}$ & $20.3 \mathrm{~b}$ & $14.0 \mathrm{~b}$ & $21.6 \mathrm{a}$ & $101.1 \mathrm{a}$ \\
\hline RRP & $12.3 \mathrm{def}$ & $8.0 \mathrm{e}$ & $1.5 \mathrm{a}$ & $15.7 \mathrm{~b}$ & $47174 \mathrm{abc}$ & $2.71 \mathrm{c}$ & 335.7 ef & $290.7 \mathrm{c}$ & $64.4 \mathrm{fg}$ & $4.0 \mathrm{f}$ & $18.0 \mathrm{de}$ & $13.0 \mathrm{c}$ & & $86.4 \mathrm{~b}$ \\
\hline $\mathrm{NqRC}$ & 10.7 efg & $7.0 \mathrm{e}$ & $1.6 \mathrm{a}$ & $22.7 \mathrm{ab}$ & $32523 \mathrm{bc}$ & 1.29 efg & $368.9 \mathrm{~cd}$ & $193.2 \mathrm{f}$ & $87.9 \mathrm{bc}$ & $4.0 \mathrm{f}$ & $23.0 \mathrm{a}$ & $14.9 \mathrm{a}$ & $16.4 \mathrm{c}$ & $64.6 \mathrm{c}$ \\
\hline NSRP & $13.7 \mathrm{cde}$ & $13.3 \mathrm{~cd}$ & $1.1 \mathrm{~cd}$ & $31.7 \mathrm{ab}$ & $35186 \mathrm{abc}$ & $1.00 \mathrm{~g}$ & $283.6 \mathrm{gh}$ & $165.6 \mathrm{~g}$ & $74.1 \mathrm{def}$ & $4.0 \mathrm{f}$ & $16.9 \mathrm{fg}$ & $7.8 \mathrm{f}$ & $16.9 \mathrm{bc}$ & $53.8 \mathrm{~d}$ \\
\hline qSC & 15 & & $9 d$ & $3 \mathrm{ab}$ & & $1.08 \mathrm{efg}$ & $9 \mathrm{~h}$ & & & $3.0 \mathrm{~g}$ & & $2.0 \mathrm{~d}$ & & $30.1 \mathrm{f}$ \\
\hline SC & 15 & $20.0 \mathrm{a}$ & & $.0 \mathrm{ab}$ & 2785 & 1.09 efg & $266.4 \mathrm{hi}$ & $7.9 \mathrm{e}$ & & $3.3 \mathrm{~g}$ & $18.8 \mathrm{~cd}$ & $2.0 \mathrm{~d}$ & & $30.3 \mathrm{f}$ \\
\hline NSRC & $17.0 \mathrm{bc}$ & $18.0 \mathrm{abc}$ & $\mathrm{cd}$ & $23.7 \mathrm{ab}$ & $51278 \mathrm{abc}$ & $1.96 \mathrm{~d}$ & & $7.1 \mathrm{f}$ & & $4.0 \mathrm{f}$ & $18.4 \mathrm{~cd}$ & $11.9 \mathrm{~d}$ & & $55.6 \mathrm{~d}$ \\
\hline DSI & $12.3 \mathrm{def}$ & $13.3 \mathrm{~cd}$ & $1.0 \mathrm{~cd}$ & $20.3 \mathrm{ab}$ & $67345 \mathrm{ab}$ & $3.19 \mathrm{~b}$ & $311.0 \mathrm{fg}$ & $3.1 \mathrm{c}$ & $96.1 \mathrm{~b}$ & $5.0 \mathrm{e}$ & $19.1 \mathrm{c}$ & $4.0 \mathrm{i}$ & & $41.1 \mathrm{e}$ \\
\hline GM & 12.9 & 13.2 & 1.1 & 22.6 & & 2.0 & 318.9 & 243.0 & 78.2 & 5.0 & 17.6 & & & 54.0 \\
\hline$P$ value & $<.001$ & $<.001$ & $<.001$ & 0.452 & 0.182 & $<.001$ & $<.001$ & $<.001$ & $<.001$ & $<.001$ & $<.001$ & $<.001$ & $<.001$ & $<.001$ \\
\hline LSD & 3.89 & 4.97 & 0.62 & 24.9 & 48197.0 & 0.42 & 3.03 & 1.58 & 1.23 & 0.41 & 0.95 & 0.54 & 1.91 & 6.31 \\
\hline $\mathrm{CV} \%$ & 18.1 & 22.6 & 34.1 & 66.0 & 66.0 & 20.8 & 9.6 & 6.6 & 15.9 & 8.4 & 5.4 & 5.7 & 12.3 & 11.7 \\
\hline
\end{tabular}

LR-Landraces. Description of landraces is in Table 1. Variables; NSF/p-Number of staminate flowers, NPF/p-Number of pistillate flowers, FSR-Flower sex ratio, NF/P-Number of fruits per plot, FY/P-Fruit yield per plot (kg/ha), FM-Fruit mass (kg), FL-Fruit length (mm), FW-Fruit width (mm), FRT-Fruit rind thickness ( $\mathrm{mm})$, PL-Pedicel length ( $\mathrm{mm})$, SL-Seed length (mm), SW-Seed width (mm), 100SM-100 Seed mass (g) and TSM-Total seed mass (g). Means within a column followed by the same letters are not significantly different at $\mathrm{p}<0.05$ according to multiple range test. 
Table 4. Correlation among agro-morphological traits of Lagenaria siceraria landraces

\begin{tabular}{|c|c|c|c|c|c|c|c|c|c|c|c|c|c|c|c|}
\hline Variables & GP & SH & CLA & SG & LG & VL & NOB & NOL & TCC & SFM & SDM & SMC & TBL & TLL & TSHL \\
\hline SH & 0.083 & & & & & & & & & & & & & & \\
\hline CLA & 0.423 & 0.532 & & & & & & & & & & & & & \\
\hline SG & -0.004 & 0.193 & 0.029 & & & & & & & & & & & & \\
\hline LG & -0.114 & 0.115 & -0.280 & 0.392 & & & & & & & & & & & \\
\hline VL & 0.217 & 0.247 & 0.296 & -0.588 & -0.327 & & & & & & & & & & \\
\hline NOB & 0.179 & -0.114 & -0.245 & -0.212 & -0.115 & 0.516 & & & & & & & & & \\
\hline NOL & -0.014 & 0.399 & 0.162 & -0.118 & 0.146 & 0.678 & 0.614 & & & & & & & & \\
\hline TCC & -0.310 & -0.089 & -0.096 & 0.194 & -0.101 & -0.346 & -0.294 & -0.270 & & & & & & & \\
\hline SFM & -0.219 & 0.296 & 0.440 & 0.461 & 0.345 & -0.501 & -0.651 & -0.144 & -0.025 & & & & & & \\
\hline SDM & -0.380 & 0.271 & 0.155 & 0.515 & 0.365 & -0.517 & -0.566 & -0.115 & -0.045 & 0.857 & & & & & \\
\hline SMC & 0.140 & 0.158 & 0.449 & 0.066 & 0.058 & -0.047 & -0.140 & 0.146 & 0.108 & 0.381 & -0.093 & & & & \\
\hline TBL & -0.728 & 0.144 & -0.235 & 0.139 & 0.267 & -0.418 & -0.361 & -0.075 & -0.021 & 0.517 & 0.701 & -0.199 & & & \\
\hline TLL & -0.280 & -0.139 & -0.046 & -0.036 & -0.060 & -0.478 & -0.438 & -0.354 & -0.205 & 0.516 & 0.551 & 0.059 & 0.653 & & \\
\hline TSHL & -0.076 & 0.129 & 0.143 & 0.072 & -0.036 & -0.403 & -0.267 & -0.292 & -0.355 & 0.513 & 0.347 & 0.364 & 0.433 & 0.775 & \\
\hline $\mathrm{NSF} / \mathrm{P}$ & -0.160 & 0.057 & 0.377 & 0.100 & -0.128 & -0.076 & 0.118 & 0.082 & 0.020 & 0.393 & 0.364 & -0.038 & 0.194 & 0.051 & 0.099 \\
\hline $\mathrm{NPF} / \mathrm{p}$ & 0.022 & 0.439 & 0.565 & 0.300 & 0.017 & 0.023 & -0.030 & 0.092 & 0.106 & 0.375 & 0.296 & 0.016 & -0.014 & -0.335 & -0.116 \\
\hline FSR & -0.152 & -0.387 & -0.303 & -0.306 & -0.203 & -0.035 & 0.089 & -0.007 & -0.022 & -0.142 & -0.209 & 0.313 & 0.032 & 0.495 & 0.411 \\
\hline $\mathrm{NF} / \mathrm{p}$ & 0.155 & 0.011 & 0.049 & -0.132 & -0.011 & 0.234 & 0.673 & 0.401 & -0.172 & -0.174 & -0.102 & -0.134 & -0.229 & -0.213 & -0.119 \\
\hline $\mathrm{FY} / \mathrm{p}$ & -0.201 & 0.124 & 0.112 & 0.256 & 0.033 & -0.296 & -0.003 & 0.140 & 0.427 & 0.317 & 0.171 & 0.392 & 0.187 & -0.066 & 0.025 \\
\hline FM & -0.293 & 0.064 & -0.019 & 0.249 & -0.007 & -0.424 & -0.403 & -0.173 & 0.368 & 0.358 & 0.231 & 0.350 & 0.356 & 0.165 & 0.163 \\
\hline FL & -0.138 & 0.307 & 0.451 & 0.297 & -0.143 & -0.002 & -0.290 & 0.062 & 0.070 & 0.390 & 0.113 & 0.565 & 0.164 & 0.113 & 0.369 \\
\hline FW & -0.430 & -0.061 & -0.165 & 0.338 & 0.238 & -0.560 & -0.510 & -0.229 & 0.411 & 0.477 & 0.423 & 0.241 & 0.425 & 0.181 & 0.064 \\
\hline PL & 0.504 & 0.132 & 0.557 & 0.030 & -0.494 & 0.409 & 0.189 & 0.133 & & -0.243 & -0.422 & 0.175 & -0.513 & -0.357 & -0.148 \\
\hline FRT & -0.176 & -0.211 & 0.505 & -0.052 & 0.141 & & 0.4 & & & & & & & & -0.395 \\
\hline SL & 140 & 0.019 & & & & & & & & & & & & & 639 \\
\hline SW & .393 & .239 & 89 & & & & & & & & & & & & 624 \\
\hline $100 \mathrm{SM}$ & 38 & $0.0^{\circ}$ & & & & & & & & & & & 4 & & 0.385 \\
\hline TSM & -0.515 & -0.045 & -0.176 & 0.155 & 0.148 & -0.367 & -0.214 & 0.036 & 0.062 & 0.412 & 0.288 & 0.426 & 0.609 & 0.446 & 0.435 \\
\hline $\mathrm{NPF} / \mathrm{p}$ & 0.781 & & & & & & & & & & & & & & \\
\hline FSR & -0.390 & -0.784 & & & & & & & & & & & & & \\
\hline $\mathrm{NF} / \mathrm{p}$ & 0.613 & 0.419 & -0.144 & & & & & & & & & & & & \\
\hline $\mathrm{FY} / \mathrm{p}$ & 0.419 & 0.314 & -0.115 & 0.082 & & & & & & & & & & & \\
\hline $\mathrm{FM}$ & -0.042 & -0.046 & 0.010 & -0.532 & 0.768 & & & & & & & & & & \\
\hline FL & -0.033 & 0.088 & 0.123 & 0.544 & 0.232 & 0.437 & & & & & & & & & \\
\hline FW & -0.010 & -0.037 & -0.025 & -0.490 & 0.702 & 0.916 & 0.254 & & & & & & & & \\
\hline PL & 0.052 & 0.169 & -0.049 & -0.062 & -0.022 & -0.124 & 0.499 & -0.371 & & & & & & & \\
\hline FRT & -0.034 & -0.077 & 0.005 & 0.189 & 0.543 & 0.364 & -0.262 & 0.4076 & -0.227 & & & & & & \\
\hline SL & 0.003 & -0.240 & 0.275 & -0.305 & 0.001 & 0.197 & 0.396 & 0.077 & 0.234 & -0.472 & & & & & \\
\hline SW & 0.203 & -0.088 & 0.217 & -0.171 & -0.053 & 0.136 & 0.312 & 0.094 & -0.129 & -0.396 & 0.637 & & & & \\
\hline $100 \mathrm{SM}$ & -0.062 & -0.153 & 0.225 & -0.419 & 0.575 & 0.780 & 0.597 & 0.665 & -0.185 & 0.247 & 0.226 & 0.351 & & & \\
\hline TSM & -0.048 & -0.244 & 0.315 & -0.439 & 0.576 & 0.799 & 0.518 & 0.732 & -0.278 & 0.206 & 0.339 & 0.461 & 0.966 & & \\
\hline
\end{tabular}

Note: Correlation is significant at the $\mathrm{P}>0.05$ level. Mean value $>0.6$ deemed to be significantly correlated. Variables are described in Tables 2 and 3

These traits represent $13.914 \%$ of the total variability. In a comparable study, fruit width strongly correlated positively with PC1 ( $\mathrm{r}=0.870)$ (Mlandenovic et al. 2012). Morimoto et al. (2005) reported; fruit width, seed; length and width strongly correlated positively with $\mathrm{PC} 1,(\mathrm{r}=0.79, \mathrm{r}=0.90$ and $\mathrm{r}=0.81$ ), respectively. Results of a comparative study with landraces from Limpopo, strongly disagree with the current findings. Vine length and number of branches correlated negatively with PC1 in the current study but positively with $\mathrm{PC} 1$ in landraces from Limpopo (Mashilo et al. 2016). In the same study by Mashilo et al. (2016) staminate and pistillate flowers correlated positively with PC1, whereas in this study pistillate flowers positively correlated with PC2 and staminate flowers had insignificant correlations with all PCs.

\section{Cluster analysis}

Lagenaria siceraria landraces clustered according to their fruit and seed morphology as well as their origin in a biplot (Figure 1) and dendrogram (Figure 2). Cluster I in the biplot and Groups I and II of the dendrogram consisted of landraces with Asiatica type seeds, that had pear-shaped (KSP, RSP, RRP) and curvilinear-shaped (NqRC) fruits that were either smooth and rough in texture (Table 1). All these landraces had shorter vines, relatively fewer leaves, fewer staminate flowers and fruits per plot (Tables 2 and 3). In addition, landraces RSP, RRP and NqRC were related based on short seedlings with small cotyledonous leaves; longer long-tendril lobes and fewer pistillate flowers per plot, which in each trait it was the opposite for landrace KSP. Clustering of $L$. siceraria landraces 
according to their fruit shape and texture concurs the findings of Morimoto et al. (2005). Findings by Kalyanrao et al. (2016), concurs clustering according to fruit shape and fruit color. Cluster II in the biplot (Figure 1) and Group IV of the dendrogram (Figure 2) grouped landraces NRB and NRC from Ndumo with rough fruit rinds and siceraria type seeds. Chimonyo and Modi (2013) reported similar findings where $L$. siceraria landraces of the same morphology and origin clustered together.

The remaining landraces formed Cluster III in a biplot and Group III of the dendrogram (Figures 1 and 2). Subgroup IIIa of the dendrogram consisted of landraces with asiatica seed type, smooth fruit rind and dominantly curvilinear-shaped fruits, but from different origins (KSC, MSC, DSI, NqSC and NSC) (Table 1; Figure 2). Similarly, L. siceraria landraces from Kenya with similar morphology and different origins clustered together (Morimoto et al. 2005). Landrace DSI had the highest germination percentage (Table 2). Landrace KSC had superior seedling height, shoot growth, leaf growth as well as shoot fresh and dry mass (Table 2 and 3). In the same Sub-group (IIIa) landraces from the same origin (Nquthu) with semicurvilinear (NqSC) and curvilinear (NSC) fruit shape, had a high similarity index (0.95) (Figure 2). Sub-group III b associated landraces with rough fruit rinds and intermediate to siceraria seed type but with different origins (KRI, NSRP and NSRC) (Table 1; Figure 2). In this Sub-group (IIIb), semi-rough textured landraces from the same origin (Nquthu) with pear- (NSRP) and curvilinear- (NSRC) shaped fruits, also had a high similarity index (0.90) (Figure 2). The clustering of landraces according to their reproductive traits from different origins agrees with the findings by Koffi et al. (2009) and Mlandenovic et al. (2012) where landraces with bigger fruit and seed sizes grouped together in isolation from landraces with smaller fruit and seed sizes.

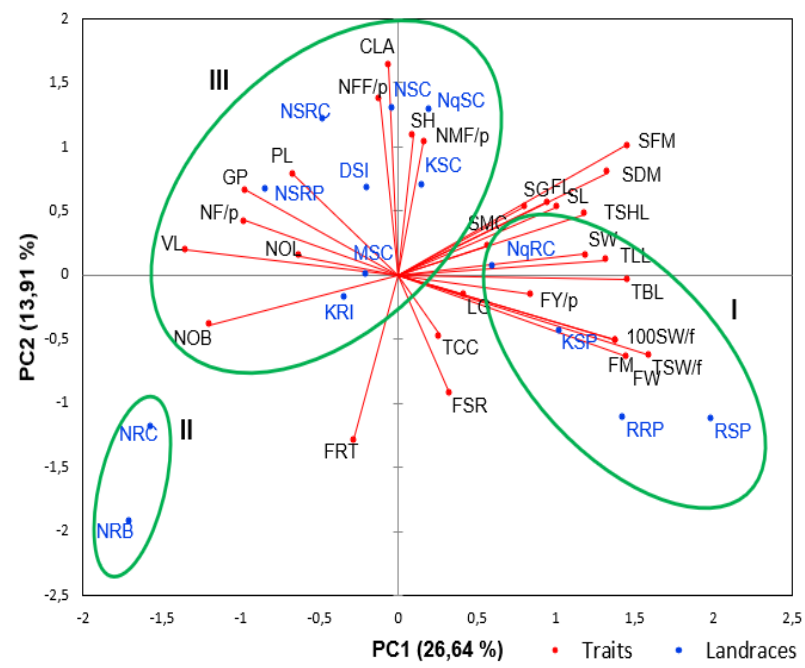

Figure 1. Biplot of Lagenaria siceraria landraces and agronomic traits. Landraces and agronomic traits (variables) are described in Table 1 and Tables 2 and 3, respectively

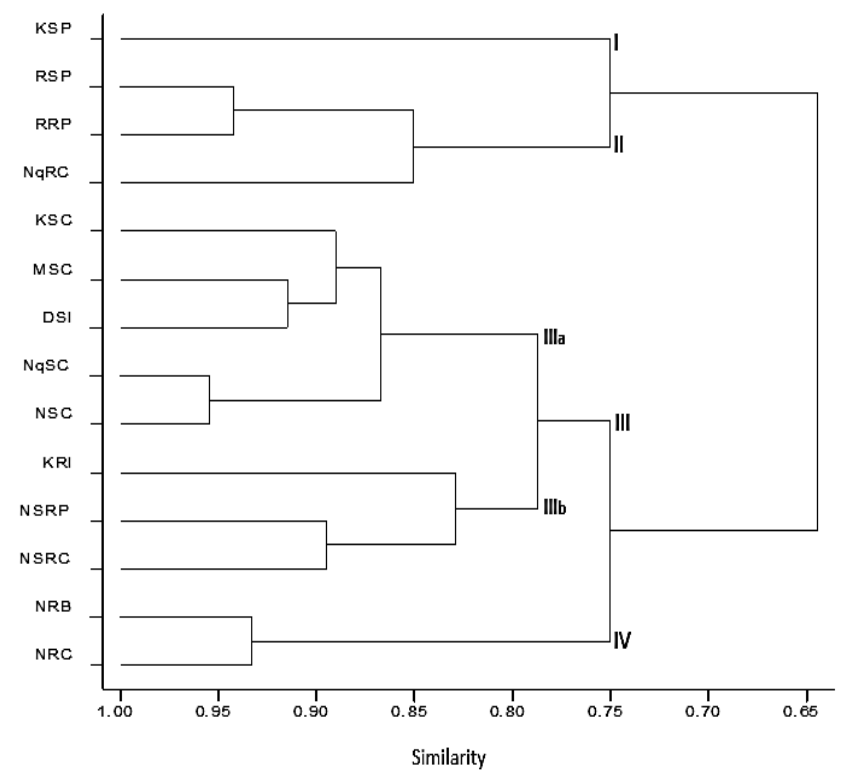

Figure 2. Hierarchical cluster showing similarities amongst Lagenaria siceraria landraces using the complete linkage method. Description of landraces is in Table 1 ..

Table 5. Loadings of the variables for the first five principal components

\begin{tabular}{|c|c|c|c|c|c|}
\hline Variables & PC1 & PC2 & PC3 & PC4 & PC5 \\
\hline GP & -0.499 & 0.340 & -0.107 & 0.316 & -0.282 \\
\hline SH & 0.049 & 0.563 & 0.309 & 0.121 & 0.120 \\
\hline CLA & -0.031 & 0.843 & 0.197 & 0.398 & -0.071 \\
\hline SG & 0.412 & 0.277 & 0.245 & -0.214 & -0.263 \\
\hline LG & 0.215 & -0.080 & 0.177 & -0.428 & 0.034 \\
\hline VL & -0.696 & 0.101 & 0.065 & 0.384 & 0.349 \\
\hline NOB & -0.620 & -0.201 & 0.087 & 0.064 & 0.625 \\
\hline NOL & -0.322 & 0.076 & 0.346 & 0.199 & 0.673 \\
\hline TCC & 0.134 & -0.243 & 0.464 & -0.064 & -0.497 \\
\hline SFM & 0.751 & 0.522 & 0.107 & -0.146 & 0.000 \\
\hline SDM & 0.685 & 0.411 & -0.026 & -0.479 & 0.041 \\
\hline SMC & 0.291 & 0.116 & 0.296 & 0.630 & 0.063 \\
\hline TBL & 0.752 & -0.018 & -0.144 & -0.346 & 0.316 \\
\hline TLL & 0.679 & 0.060 & -0.627 & -0.047 & 0.172 \\
\hline TSHL & 0.608 & 0.243 & -0.464 & 0.198 & 0.266 \\
\hline $\mathrm{NSF} / \mathrm{P}$ & 0.082 & 0.532 & 0.301 & -0.362 & 0.378 \\
\hline $\mathrm{NFF} / \mathrm{p}$ & -0.060 & 0.706 & 0.542 & -0.313 & 0.046 \\
\hline FSR & 0.166 & -0.473 & -0.489 & 0.431 & 0.212 \\
\hline $\mathrm{NF} / \mathrm{p}$ & -0.505 & 0.219 & 0.131 & -0.392 & 0.552 \\
\hline $\mathrm{FY} / \mathrm{p}$ & 0.436 & -0.076 & 0.752 & 0.091 & 0.176 \\
\hline FM & 0.713 & -0.264 & 0.481 & 0.258 & -0.129 \\
\hline FL & 0.490 & 0.294 & 0.135 & 0.677 & -0.030 \\
\hline FW & 0.745 & -0.327 & 0.483 & -0.038 & -0.193 \\
\hline PL & -0.346 & 0.405 & 0.060 & 0.641 & -0.196 \\
\hline FRT & -0.144 & -0.660 & 0.613 & -0.075 & 0.166 \\
\hline SL & 0.524 & 0.274 & -0.510 & 0.238 & 0.055 \\
\hline SW & 0.612 & 0.082 & -0.494 & -0.094 & 0.220 \\
\hline 100SM & 0.711 & -0.262 & 0.289 & 0.397 & 0.340 \\
\hline TSM & 0.820 & -0.321 & 0.178 & 0.278 & 0.298 \\
\hline Eigenvalue & 7.724 & 4.035 & 3.999 & 3.325 & 2.490 \\
\hline Variability $\%$ & 26.635 & 13.914 & 13.791 & 11.465 & 8.587 \\
\hline Cumulative $\%$ & 26.635 & 40.550 & 54.340 & 65.806 & 74.393 \\
\hline
\end{tabular}

Note: PC1-5: Principal components 1-5. Variables are described in Tables 2 and 3 
Table 6. Genetic parameters for agronomic traits of Lagenaria siceraria landraces

\begin{tabular}{|c|c|c|c|c|c|c|c|c|c|}
\hline Variables & $\delta^{2} \mathrm{~g}$ & $\delta^{2} e$ & $\delta^{2} p$ & GM & PCV & GCV & ECV\% & H2 & GA \\
\hline GP & 2.1 & 69.3 & 71.4 & 70.5 & 10.1 & 1.7 & 9.9 & 3.0 & 0.4 \\
\hline $\mathrm{SH}$ & 46.1 & 24.1 & 70.2 & 37.4 & 13.7 & 11.1 & 8.0 & 65.7 & 1.7 \\
\hline CLA & 72300 & 73251 & 145552 & 1289 & 106.3 & 74.9 & 75.4 & 49.7 & 1.5 \\
\hline SG & 195.9 & 2319 & 2514.9 & 88.9 & 53.2 & 14.8 & 51.1 & 7.8 & 0.6 \\
\hline LG & 3185028 & 3533042 & 6718070 & 5231 & 246.8 & 259.9 & 259.9 & 47.4 & 1.4 \\
\hline VL & 0.3 & 0.3 & 0.6 & 2.9 & 4.6 & 3.3 & 3.2 & 52.2 & 1.5 \\
\hline NOB & 2.1 & 0.5 & 2.6 & 6.1 & 6.6 & 5.8 & 2.9 & 79.6 & 1.8 \\
\hline NOL & 52.2 & 19.8 & 72.0 & 29.7 & 15.6 & 13.3 & 8.2 & 72.5 & 1.8 \\
\hline TCC & 7.8E-07 & $1.2 \mathrm{E}-05$ & $1.3 \mathrm{E}-05$ & 0.01 & 0.3 & 0.1 & 0.3 & 6.0 & 0.5 \\
\hline SFM & 56.3 & 70.3 & 126.6 & 53.9 & 15.3 & 10.2 & 11.4 & 44.5 & 1.4 \\
\hline SDM & 1.9 & 71.3 & 73.2 & 54.9 & 11.6 & 1.9 & 11.4 & 2.7 & 0.3 \\
\hline SMC & 0.1 & 72.3 & 72.3 & 55.9 & 11.4 & 0.3 & 11.4 & 0.1 & 0.1 \\
\hline TBL & 116.4 & 86.5 & 202.9 & 53.3 & 19.5 & 14.8 & 12.7 & 57.4 & 1.6 \\
\hline TLL & 1140 & 1129 & 2269 & 211.2 & 32.8 & 23.2 & 23.1 & 50.2 & 1.5 \\
\hline TSHL & 363.6 & 405.4 & 769.0 & 125.4 & 24.8 & 17.0 & 17.9 & 47.3 & 1.4 \\
\hline $\mathrm{NSF} / \mathrm{P}$ & 14.6 & 73.3 & 87.9 & 56.9 & 12.4 & 5.0 & 11.4 & 16.6 & 0.8 \\
\hline $\mathrm{NFF} / \mathrm{p}$ & 20.9 & 74.3 & 95.2 & 57.9 & 12.8 & 6.0 & 11.3 & 21.9 & 0.9 \\
\hline FSR & 3.8 & 75.3 & 79.0 & 58.9 & 11.9 & 2.5 & 11.3 & 4.8 & 0.4 \\
\hline $\mathrm{NF} / \mathrm{p}$ & 2.3 & 76.3 & 78.6 & 59.9 & 11.5 & 1.9 & 11.3 & 2.9 & 0.4 \\
\hline $\mathrm{FY} / \mathrm{p}$ & $1.36 \mathrm{E}+08$ & 77.3 & $1.36 \mathrm{E}+08$ & 60.9 & 14959.7 & 14959.7 & 11.3 & 99.9 & 2.1 \\
\hline FM & 2.0 & 0.2 & 2.2 & 2.0 & 10.5 & 10.0 & 2.95 & 91.9 & 2.0 \\
\hline FL & 55.9 & 9.3 & 65.3 & 31.9 & 14.3 & 13.2 & 5.4 & 85.7 & 1.9 \\
\hline FW & 37.8 & 2.6 & 40.4 & 24.3 & 12.9 & 12.5 & 3.2 & 93.7 & 2.0 \\
\hline PL & $\begin{array}{r}1.9 \\
1.9\end{array}$ & 1.6 & 3.5 & 7.8 & 6.7 & 4.9 & 4.5 & 55.1 & 1.5 \\
\hline FRT & 2.7 & 0.2 & 2.8 & 5.0 & 7.5 & 7.3 & 1.9 & 93.8 & 2.0 \\
\hline SL & 4.9 & 0.9 & 5.8 & 17.6 & 5.7 & 5.3 & 2.3 & 84.1 & 1.9 \\
\hline SW & 10.3 & 0.3 & 10.6 & 9.7 & 10.4 & 10.3 & 1.8 & 97.1 & 2.0 \\
\hline $100 \mathrm{SM}$ & 10.7 & 3.7 & 14.4 & 15.6 & 9.6 & 8.3 & 4.9 & 74.2 & 1.8 \\
\hline TSM & 535.2 & 40.4 & 575.6 & 54.1 & 32.6 & 31.5 & 8.6 & 92.9 & 2.0 \\
\hline
\end{tabular}

Note: $\delta^{2}$ g-genotypic variance, $\delta^{2}$ e-environmental variance, $\delta^{2}$ p-phenotypic variance, GCV-genotypic coefficient of variation, PCVphenotypic coefficient of variation, EVC\%-environmental coefficient of variation, H2-broad sense heritability, GA-genetic advancement. Variables (agronomic traits) are explained in Tables 2 and 3.

\section{Genetic parameters}

The phenotypic coefficient was generally higher than the genotypic coefficient of variability indicating a strong impact of the environment towards the expressiveness of genes in the phenotypic display (Table 6). Jain et al. (2017) recorded similar results. High to a moderate coefficient of variation was recorded in the current study for fruit yield per plot $(14959.7 \%)$, leaf growth $(246.8 \%)$, cotyledonous leaf area $(106.3 \%)$, tendril longer lobe length $(32.8 \%)$ and total seed mass $(32.6 \%)$. Similar results were recorded by Singh et al. (2015), fruit mass (1303.33\%) and yield (373.29 \%). Yadagiri et al. (2017) recorded similar phenotypic coefficient results, fruit mass at $(39.35 \%)$ and yield at $(71.79 \%)$. The highest value for broad sense estimate was recorded for the following traits; fruit yield per plot (99.9), seed width (97.1), fruit rind thickness (93.8), fruit width (93.7), total seed mass (92.9), fruit mass (91.9), fruit length (85.7), seed length (84.1), number of branches (79.6), 100 seed mass (74.2) and number of leaves (72.5). Yadagiri et al. (2017) recorded similar broad sense heritability results; yield (99.70), seed width (83.60), fruit length (91.40) and number of branches (76.70).

The genetic advance of the current study was lower for all traits when compared to studies conducted by Jain et al.
(2017) and Yadagiri et al. (2017). The current study had higher genetic advancement compared to landraces studied by Rani and Reddy (2017).

In conclusion, curvilinear-shaped landraces (KSC, MSC, NqRC, NqSC, NSC, and NSRC) out-performed all landraces through vigorous growth of seedling and vegetative traits. These landraces are recommended for genetic improvement upon artificial selection. Landraces with large-sized fruits (KSP, RSP, and DSI) produced fewer numbers of fruits compared with landraces with small-sized fruits (KRI, NSRP and NqSC). Fruit and seed morphology, as well as the area of origin, were the most determining factors to clustering together of landraces with similar phenotypic traits. Fruit and seed traits have very high heritability estimates coupled with high genetic advancement. This is indicative of the additive gene action and possible genetic gain upon selection and preservation. 


\section{ACKNOWLEDGEMENTS}

This work was financially supported by the Department of Agriculture, Forestry and Fisheries and the University of Zululand, South Africa postgraduate bursary scheme.

\section{REFERENCES}

Allard RW. 1960. Principles of Plant Breeding. John Wiley and Sons, London.

Burton GW, Devane EH. 1953. Estimating the heritability in tall fescue (Festuca arundinancea) from replicated clonal material. J Agron 45: 478-481.

Chimonyo VGP, Modi AT. 2013. Seed performance of selected bottle gourd (Lagenaria siceraria (Molina) Standl). Amer J Exp Agric 3 (4) 740-766.

Deepthi B, Reddy PSS, Kumar AS, Reddy AR. 2016. Studies on $p c v, g c v$ heritability and genetic advance in bottle gourd genotypes for yield and yield components. Plant Arch 16 (2): 597-601.

Jain A, Singh SP, Pandey VP. 2017. Character association among the yield and yield attributes in bottle gourd [Lagenaria siceraria (Molina) Standl] genotypes. Plant Arch 17 (1): 711-714

Kalyanrao P, Tomar BS, Singh B, Aher B. 2016. Morphological characterization of parental lines and cultivated genotypes of bottle gourd (Lagenaria siceraria). Indian J Agric Sci 86 (1): 65-70.

Koffi KK, Anzara GK, Malice M, Dje Y, Bertin P, Baudoin JP, ZoroBi IA. 2009. Morphological and allozyme variation in a collection of Lagenaria siceraria (Mol.) standl. From Cote d'Iviore. Biotechnol Agron Soc Environ 13 (2): 257-270.

Koppad SB, Chavan M, Hallur R. 2016.Character association studies an path coefficient analysis for yield and yield attributing traits in Ridge gourd [Luffa acutangula (L.) Roxb.]. Electronic J Plant Breed 7 (2): 275-281.

Korkmaz A, Dufault RJ. 2001. Developmental consequences of cold temperature stress at transplanting on seedling and field growth and yield. i. watermelon. J Amer Soc Hort Sci 126 (4): 404-409.

Mashilo J, Shimelis H, Odindo A. 2016. Genetic diversity of bottle gourd (Lagenaria siceraria (Molina) Standl.) landraces of South Africa assessed by morphological traits and simple sequence repeat markers. S A J Plant Soil 33 (2): 113-124.

Mashilo J, Shimelis H, Odindo A. 2017. Phenotypic and genotypic characterization of bottle gourd [Lagenaria siceraria (Molina) Standl.] and implications for breeding: A Review. Scientia Horticulturae 222: 136-144

Meena BL, Das SP, Meena SK, Kumari R, Devi AG, Devi HL. 2015. Assessment of GCV, PCV, heritability and genetic advance for yield and its components in field pea (PisumsativumL.). Intl J Curr Microbiol Appl Sci 6 (5): 1025-1033.

Mlandenovic E, Berenji J, Ognjanov V, Kraljevic-Balalic M, Ljubojevic M, Cukanovic M. 2011. Conservation and morphological characterization of bottle gourd for ornamental use. Faculty of Agriculture, University of Nov Sad, Serbia.

Mlandenovic E, Berenji J, Ognjanov V, Ljubojevic M, Cukanovic J. 2012. Genetic variability of bottle gourd Lagenaria siceraria (Mol.) Standley and its morphological characterization by multivariate analysis. Arch Biol Sci 6 (2): 573-583.

Mohammed AS, Salem EA, Helaly AA. 2017. Impact of mycorrhizae and polyethylene mulching on growth, yield and seed oil production of bottle gourd (Lagenaria siceraria). J Hortic Sci Ornamental Plants 9 (1): 28-38.

Morimoto Y, Maundu P, Fujumaki H, Morishuma H. 2005. Diversity of landraces of the white-flowered gourd (Lagenaria siceraria) and its wild relatives in Kenya: fruit and seed morphology. Genet Res Crop Evol 52: 737-747.

Muralidharan B, Kanthaswamy V, Janaranjani KG. 2017. Correlation and path analysis for quantitative and qualitative traits in bottle gourd [Lagenaria siceraria (Molina) standl]. J Pharmacogn Phytochem 6 (5): 2280-2283.

Ntuli NR, Zobolo AM. 2008. Effect of water stress on growth of colchicine-induced polyploid Coccinia palmata and Lagenaria sphaerica plants. African J Biotechnol 7 (20): 3548-3652.

Ntuli NR, Madakadze RM, Zobolo AM. 2017. Variation in morphology and yield traits of Cucurbita landraces in northern KwaZulu-Natal, South Africa. S A J Plant Soil 34 (5): 389-397.

Rani KU, Reddy EN. 2017. Variability and correlation studies in bottle gourd. International J Pure and Applied Biosciences 5 (2): 723-731

Sharma RK, Tomar BS. 2016. Influence of growing direction on seed yield and quality in bottle gourd (Lagenaria siceraria). Indian J Agric Sci 86 (7): 895-900.

Singh RP, Jain PK, Gontia AS, Verma AK. 2017. Physiological evaluation of different genotypes and their F1 progenies in bottle gourd [Lagenaria siceraria (Molina) Standley]. Intl J Chem Stud 5 (3): 74-76.

Singh SP, Singh B, Mohan M, Rao S, Soni S. 2014.Genetic variability for some quantitative traits in bottle gourd [Lagenaria siceraria (Molina) Standl.]. Ann Hortic 8 (1): 113-115.

Sivaraj N, Pandravada SR. 2005. Morphological diversity for fruit characters in bottle gourd Germplasm from tribal pockets of Telangana region of Andhra Pradesh, India. Asian Agri-History 9 (4): 305-310.

Uddin AFMJ, Tahidul MI, Chowdhury MHN, Shiam IM, Mehraj H. 2014. Evaluation of bottle gourd (Lagenaria siceraria) to growth and yield. International J Biosci 5 (12): 7-11.

Xaba P, Croeser P. 2011. The calabash gourd. Kirstenbosch National Botanical Garden. Veld and Flora, South Africa.

Yadagiri J, Gupta NK, Tembhire D, Sheela V. 2017. Genetic variability, heritability and morphological characterization in bitter gourd (Momordica charatia L.). Intl J Pure Appl Biosci 5 (4): 1322-1327.

Yetisir H, Sakar M, Serce S. 2008. Collection and morphological characterization of Lagenaria siceraria germplasm from the Mediterranean region of Turkey. Genet Res Crop Evol 55: 12571266 